\title{
Zebrafish as a Genetic Model in Biological and Behavioral Gerontology: Where Development Meets Aging in Vertebrates - A Mini-Review
}

\author{
Shuji Kishi ${ }^{a, b}$ Barbara E. Slack ${ }^{c}$ Junzo Uchiyamad, e Irina V. Zhdanova ${ }^{f}$ \\ a Department of Metabolism and Aging, The Scripps Research Institute, ${ }^{\mathrm{b}}$ Department of Ophthalmology, \\ Harvard Medical School, 'Department of Pathology and Laboratory Medicine, Boston University School of \\ Medicine, ${ }^{\mathrm{d}}$ Department of Pathology, Harvard Medical School, e'Division of Internal Medicine, Aiseikai Clinic, and \\ fDepartment of Anatomy and Neurobiology, Boston University School of Medicine, Boston, Mass., USA
}

\section{Key Words}

Zebrafish • Early biomarkers $\cdot \beta$-galactosidase $\cdot$

Aging phenotypes later in life

\begin{abstract}
Understanding the molecular mechanisms of aging in vertebrates is a major challenge of modern biology and biomedical science. This is due, in part, to the complexity of the aging process and its multifactorial nature, the paucity of animal models that lend themselves to unbiased highthroughput screening for aging phenotypes, and the difficulty of predicting such phenotypes at an early age. We suggest that the zebrafish genetic model offers a unique opportunity to fill in this gap and contributes to advances in biological and behavioral gerontology. Our recent studies demonstrated that this diurnal vertebrate with gradual senescence is an excellent model in which to study age-dependent changes in musculoskeletal and eye morphology, endocrine factors, gene expression, circadian clock, sleep and cognitive functions. Importantly, we have also found that the presence of a senescence-associated biomarker ('senescence-associated $\beta$-galactosidase') can be documented during early zebrafish development and is predictive of premature aging phenotypes later in adult life. The availability
\end{abstract}

of mutant 'genotypes' with identified aging 'phenotypes' in zebrafish, in combination with a wealth of information about zebrafish development and genetics, and the existence of multiple mutant and transgenic lines, should significantly facilitate the use of this outstanding vertebrate model in deciphering the mechanisms of aging, and in developing preventive and therapeutic strategies to prolong the productive life span ('health span') in humans.

Copyright $\odot 2009$ S. Karger AG, Base

\section{Introduction}

The molecular mechanisms of aging are the focus of extensive investigations throughout the world, providing hope for the discovery of the principles that govern this ubiquitous process, and novel ways to attenuate or delay it in humans. Considering that humans are diurnal mammals with gradual senescence and a relatively long life span, the choice of animal models to study the mechanisms of human aging remains one of the most important issues. All the existing models of human aging have certain limitations, calling for an integrative approach that uses diverse species in the hope that each would provide a piece of the puzzle and, together, would help to identify

\section{KARGER}

Fax +4161306 1234

E-Mail karger@karger.ch

www.karger.com (c) 2009 S. Karger AG, Basel

0304-324X/09/0554-0430\$26.00/0

Accessible online at:

www.karger.com/ger
Shuji Kishi, MD, PhD

Department of Metabolism and Aging

The Scripps Research Institute, Scripps Florida

130 Scripps Way \#3B3, Jupiter, FL 33458 (USA)

Tel. +1 561228 2959, Fax +1 561228 3059, E-Mail kishi@scripps.edu 
critical elements common to aging in all organisms. This explains why current biomedical investigations into the mechanisms of aging are conducted concurrently in such phylogenetically diverse species as worms, insects, mice and non-human primates. The use of highly advanced species phylogenetically close to humans, for example rhesus monkeys, allows for critical evaluation of mechanisms of physiological and cognitive aging that might be specific to primates [1-4]. In contrast, small and prolific organisms, such as Caenorhabditis elegans and Drosophila melanogaster, provide the basis for unbiased screens that identify and determine the precise functions of novel genes that regulate aging and life span. This approach has already been successfully utilized to identify key genes evolutionarily conserved in, and associated with, the aging process. However, these 'invertebrate models', with their short life span, might not allow for the discovery of critical longevity- and senescence-associated genes unique to long-living vertebrates.

Thus far, only mice have served as a popular vertebrate genetic model system to address specific questions relevant to the aging process. The research in mice provided important insights into mammalian aging and will continue to be indispensable in studying vertebrate aging [5]. However, having only one genetic vertebrate model might be limiting, especially in view of apparent caveats. For example, there are important ecological and physiological differences between diurnal and nocturnal species. Although the neuronal, endocrine and molecular arms of the circadian clock, a major mechanism of internal synchronization, show night or day in a similar way in nocturnal mice and diurnal humans [6], these manifestations of the clock mechanism carry opposite downstream signals. Active 'ticking' of the neuronal 'master clock' during the daytime corresponds to peak locomotor and cognitive activity in humans, while associated with sleep in mice. In contrast, nighttime melatonin production or expression of core clock proteins (such as BMAL1 and CLOCK) is associated with sleep in humans but active movement, social interactions and foraging in mice. These differences are important because the molecular components of the circadian clock, in addition to showing time per se, play critical roles as regulatory proteins for multiple intracellular processes, serving as transcription factors for diverse genes [7, 8], and acting as direct modulators of epigenetic processes including chromatin remodeling [9]. Accordingly, mutations in clock genes were found to lead to premature aging in diurnal insects and nocturnal mammals [10]. However, the exact pathways by which these clock mechanisms affect aging might depend on the diurnal or nocturnal adaptation of the species. Thus, translational research on the role of circadian factors in aging should benefit from using diurnal animal models. This and other examples suggest that in order to address the many biological questions regarding vertebrate aging as well as development in a more tractable and high-throughput setting, it is crucial to develop alternative vertebrate model systems.

\section{Zebrafish as a New Vertebrate Animal Model of Aging}

Recently, the zebrafish (Danio rerio) has emerged as a highly promising model for studies of vertebrate aging [11-16]. Combined with their optical transparency at embryonic and larval stages, the small size and high fecundity of zebrafish has made them a favorite vertebrate of developmental biologists. This has resulted in detailed characterization of the zebrafish genome, the development of multiple mutant and transgenic phenotypes, and the emergence of molecular genetics techniques (e.g. targeted gene knockdown using morpholino antisense oligos) allowing for investigation of the intrinsic mechanisms underlying normal physiological and pathological events in this organism [17]. Consequently, of the vertebrates studied to date, high-throughput screens using robotic systems for evaluating mutants, identifying genes, and testing chemicals, can be performed more readily and cost-effectively in zebrafish than in any other vertebrate organism.

Zebrafish live for approximately 3 years on average and over 5 years maximally in laboratory conditions and show gradual senescence similar to humans. With age, they often display spinal curvature that is possibly related to muscle abnormalities [13]. In aging zebrafish, we detected senescence-associated $\beta$-galactosidase (SA- $\beta$-gal) activity in skin, and oxidized protein accumulation in muscle $[11,16,18]$. Aged zebrafish also demonstrate increased accrual of lipofuscin ('aging pigment') in the liver (fig. 1) [18], similar to that reported in mice and humans $[19,20]$. Intriguingly, by 4 years of age, the liver lipofuscin levels become markedly higher than those at 1 year of age [18]. Aged fish also often develop lipofuscin accumulation and drusen-like lesions in retinal pigment epithelium (RPE), similar to that seen in age-related macular degeneration (AMD) in humans. Moreover, almost all aged zebrafish develop cataracts by 4 years of age [pers. unpubl. observation], and the majority of old fish show retinal atrophy [18]. Our studies further showed several 


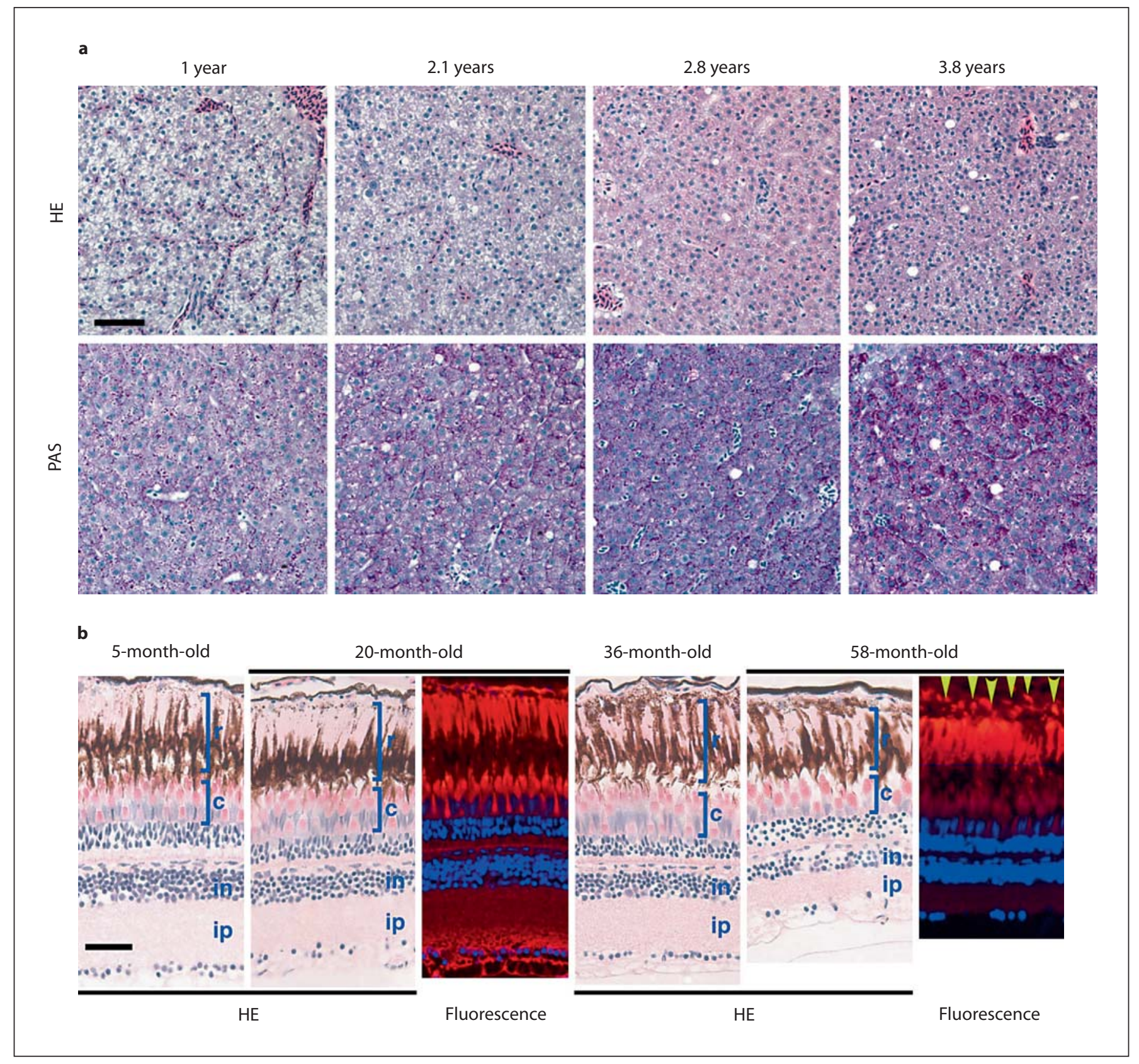

Fig. 1. Liver and eye histology in the normal adult zebrafish with age. a Liver sections of 1-, 2.1-, 2.8-, and 3.8-year wild-type zebrafish were stained with HE or PAS. Hepatocyte density and eosinophilic staining can be seen to increase with age by HE staining. PAS-positive staining for lipofuscin also shows increased levels of this biomarker in aging liver tissue. b Eye sections of 5-, 20-, 36-, and 58-month-old wild-type fish were stained with HE. In the light-adapted retina, the rods (r) sit distally to the cones (c); 'in' indicates the inner nuclear layer, and 'ip' indicates the inner plexiform layer. Processes from the pigment epithelium (PE) extend between the outer segments of the rods. Aged (58-monthold) wild-type fish show increased drusen-like accruals (yellow arrows) with autofluorescence in the RPE (lower right panel), compared with younger (20-month-old) wild-type fish (upper right panel). Scale bar: $100 \mu \mathrm{m}$ [18]. 
Fig. 2. Aging and fin regeneration in zebrafish. Representative images of caudal fin regeneration states. a During normal regeneration (within 2 weeks), the tail is morphologically reproduced in size and shape, but may still lack complete pigmentation. b In somewhat impaired regeneration, the shape remains distorted, particularly at the distal tail end. c Severely impaired fin regeneration results in segments along the incision that exhibit no regenerative capacity, as indicated by the small black arrows. Large black arrows in a-c indicate amputated regions of the caudal fins [14].

Fig. 3. Example of the circadian patterns of activity in constant dim light in young (1year-old) and aged (4-year-old) zebrafish. a, b Number of inactivity bouts (/15 min). c, d Inactivity duration ( $\mathrm{s} / 15 \mathrm{~min})$. e, $\mathbf{f}$ Distance traveled $(\mathrm{m} / 15 \mathrm{~min})$ in young and aged fish, respectively, over a 33-hour period after being maintained in $\mathrm{dl}$ for 2 days. Gray area of each plot corresponds to night period in LD [14].
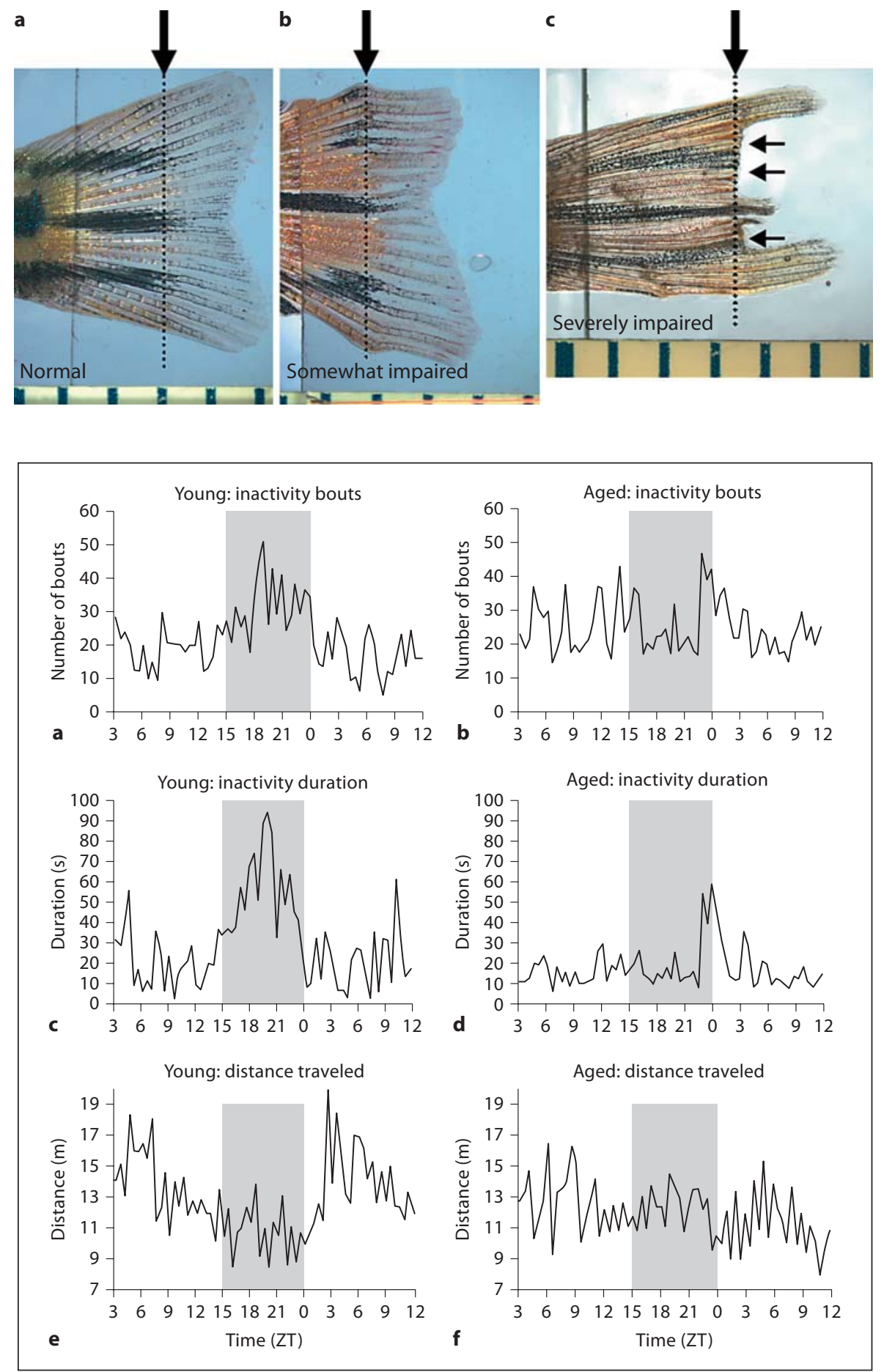

age-related degenerative changes and increases in various pathological lesions in aging zebrafish, as well as age-dependent declines in their reproductive and regenerative capacity $[11,16,18]$. In adult zebrafish, fin regeneration is a highly orchestrated process involving wound healing, establishment of the wound epithelium, recruitment of the blastema from mesenchymal cells underlying the wound epithelium, and differentiation and outgrowth of the regenerated cells [21]. Such a regenerative ability declines with age, leading to impaired morphology and dis- 
torted fin shape (fig. 2) [16]. Whereas severely impaired fin regeneration is not seen in younger fish (18 months of age), it is observed in a significant number $(33 \%)$ of older fish (30 months of age). Moreover, zebrafish exhibit ageassociated declines in the heat shock response [22, 23], and show upregulation of genes that respond to oxidative stress when subjected to life-span-increasing reductions in ambient temperature [24]. Similarities in the heat shock and oxidative stress responses between zebrafish and humans underscore the usefulness of these animals for studies on aging.

Following the initial behavioral characterization of the sleep process in zebrafish and its modulation by melatonin, which we conducted in larvae [25], we extensively investigated changes in sleep and circadian rhythms in aged zebrafish $[14,26]$. We found that in spite of the relatively long life span of this vertebrate, and its gradual senescence, sleep and circadian rhythmicity changes occur relatively early in life (fig. 3). They can be documented starting at 2 years of age and are associated with the gradual onset of melatonin deficiency and a decline in the expression of core clock genes $[14,16]$. An especially prominent decline was found in bmal1 mRNA abundance in aged zebrafish. Notably, deficiencies or mutations in this gene are associated with a premature aging phenotype in mice and Drosophila [10].

While characterizing age-dependent changes in cognitive performance in adult zebrafish, we found significant declines in learning and memory within a 4-year life span (fig. 4) [15]. A number of neurotransmitter receptors, and their downstream signaling partners, have been implicated in mechanisms underlying learning and memory. Transcriptional profiling of human brain demonstrates that many of the genes encoding these signaling molecules are downregulated in the aged brain [27], suggesting that cognitive deficits associated with aging may be a result, in part, of decreased expression of proteins important for acquisition, storage, and retrieval of memory. Our study comparing young and middle-aged wildtype zebrafish and mutants with altered acetylcholinesteraze activity (achesb55/+ mutant) suggested that the cholinergic system is an important player in zebrafish aging and affects their cognitive performance [15]. This result is especially important in view of the role the cholinergic system plays in the deficiencies associated with Alzheimer's disease [28, 29].

Overall, the age-related degenerative changes, appearance of pathological lesions, declines in reproductive and regenerative capacity, and alterations in circadian rhythmicity, sleep and cognitive function in zebrafish, suggest

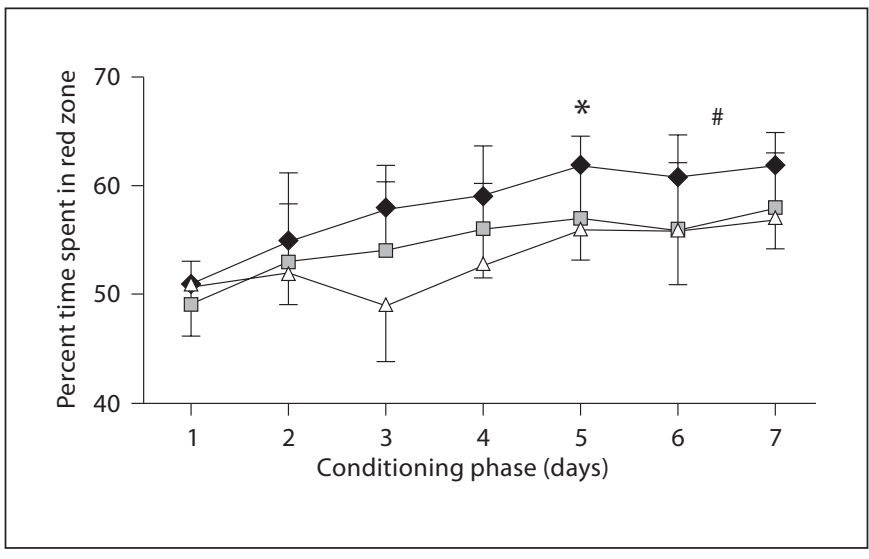

Fig. 4. Learning curves in wild-type fish of different ages. Increase in time spent in the red during $5 \mathrm{~min}$ of color presentation before food administration, compared to the same group behavior at day 1. Young fish (1-year-old) - diamond, middle-aged (2year-old) fish - square, old fish (3-year-old) - triangle. First day of significant change in group behavior: ${ }^{*} \mathrm{p}<0.01$ in young fish; ${ }^{\#} \mathrm{p}<0.05$ in middle-aged fish [15].

that these animals could be useful for explorations of the mechanisms underlying gradual senescence in vertebrates, and could serve as a promising model of age-dependent declines in integrative functions in humans [11, $14-16,18]$.

\section{Unbiased Mutant Screens for Aging Phenotypes in Vertebrates}

In addition to studying the function of specific genes that have already been linked to the aging process, it is critically important to conduct unbiased mutant screens for aging phenotypes in vertebrates, in search of additional genes that affect late-onset diseases or age-related dysfunctions. The mechanisms connecting the organismal aging process with multiple chronic human diseases ('geriatric diseases') remain a complex enigma, and scientists sometimes face conflicting arguments about 'aging phenotypes' versus 'diseases'. Both intrinsic genegene interactions ('epistasis') and environmental factors, as well as gene-environment interactions [30, 31], affect the aging process and disease progression in higher organisms like vertebrates. It is poorly understood which complex genetic elements determine life span, how these are correlated with disease susceptibility, and how environmental and epigenetic modulations affect senescence and disease development in vertebrates. There may be 
complex interactions between unrelated and/or unexpected genes, requiring unbiased methods of detection. In most vertebrates, unbiased screens for aging mutants are technically difficult and have not actually been done, even in mice. On the other hand, unbiased forward genetic screens for multiple biological and biomedical purposes have already been successfully carried out in zebrafish, and will be readily adaptable for aging research, once adequate biomarkers are identified and applicable.

The features that recommend zebrafish for such largescale screens include their small body size and high reproductive rate, allowing the evaluation of many zebrafish siblings throughout their lives under controlled conditions, with and without certain environmental and pharmacological challenges. Moreover, the existence of various zebrafish mutants and traceable transgenic phenotypes, including retroviral insertional mutant collections [32], provide a fruitful resource for the identification of both physiological and pathological aging characteristics.

\section{Utility of Early Biomarkers of Aging to Predict Aging Phenotypes Later in Life}

Humans develop and age very gradually, compared to all the models currently used for biomedical research. This might be a critical limitation, since some of the aging processes common to humans may, indeed, require a prolonged life span to become apparent, and short-span models might not be adequate to reveal determining factors. On the other hand, the lengthy life span of animals can be an obstacle to the conduct of unbiased highthroughput screens for 'potential/putative' aging mutants.

One of the promising approaches to confronting this dilemma is to assess in young animals (even embryos) reliable and easily measurable biomarkers of aging/senescence that are likely to 'predict' an aging phenotype later in life. In genotypes susceptible to accelerated (or even delayed) aging, such biomarkers might manifest in young animals with certain gene mutations, spontaneously or following stress (fig. 5). Even if such mutations are lethal in homozygous zebrafish, most heterozygous animals develop normally and usually survive into adulthood. Under normal conditions, the heterozygous embryos, larvae, or young fish might not immediately reveal significant changes in biomarkers of aging, due to the presence of at least partial compensatory mechanisms and/or adaptive regulations (e.g. hyperactivation) by a remained single allele. However, as confirmed by our recent study [18], homozygous conditions as well as exposures of these heterozygotes to specific challenges can help to amplify the response in animals showing dominant premature aging ('embryonic senescence') phenotypes even in early development. This applies to cases where gene mutation leads to an augmented response to stress or resistance to it. Thus, a mutant phenotype in homozygosity as well as a specific stress challenge in heterozygosity during early development could mimic the actual aging process and help to predict the genotypes that might be susceptible (or resistant) to premature aging.

One obvious candidate biomarker of aging to use in an unbiased screen is SA- $\beta$-gal, an indicator of cellular senescence in vitro as well as of organismal aging in vertebrates [33-38]. In fact, we detected SA- $\beta$-gal activity in skin as well as oxidized protein accumulation in muscle in aging zebrafish, similar to that shown in humans [11, $16,18]$.

We employed this marker in an unbiased screen for aging/senescence phenotypes using 306 retrovirus-mediated insertional zebrafish mutant lines $[18,32]$. Since all of the 306 mutations screened are ultimately homozygous lethal, we needed to explore the effect of missing just one copy of the genes ('haploinsufficiency') in heterozygous adult fish with age. However, instead of characterizing their aging phenotypes throughout their life span, we first examined which of these mutants showed increased SA- $\beta$-gal activity during embryonic development within 5 days after fertilization, either spontaneously in homozygote or following oxidative stress in heterozygote [18]. From the pool of 306 such mutants, we identified 11 candidates that showed higher embryonic SA- $\beta$-gal activity (fig. 6), and raised two of the lines to maturity. One of these mutants is null for a homologue of Drosophila spinster (spin/nrs), a gene known to regulate life span in flies, whereas the other harbors a mutation in a homologue of the human telomeric repeat binding factor 2 (terf2) gene, which plays roles in telomere protection and telomere-length regulation. Importantly, these two genes have been already linked to the organismal aging process and cellular senescence [39-41]. This approach allowed us to establish that prominent increases in SA- $\beta$-gal activity early in development correlate with an accelerated aging phenotype in these two mutants, with heterozygous fish showing an enhanced accumulation of lipofuscin granules, increased SA- $\beta$-gal activity, and shorter life span, compared with their control siblings. 


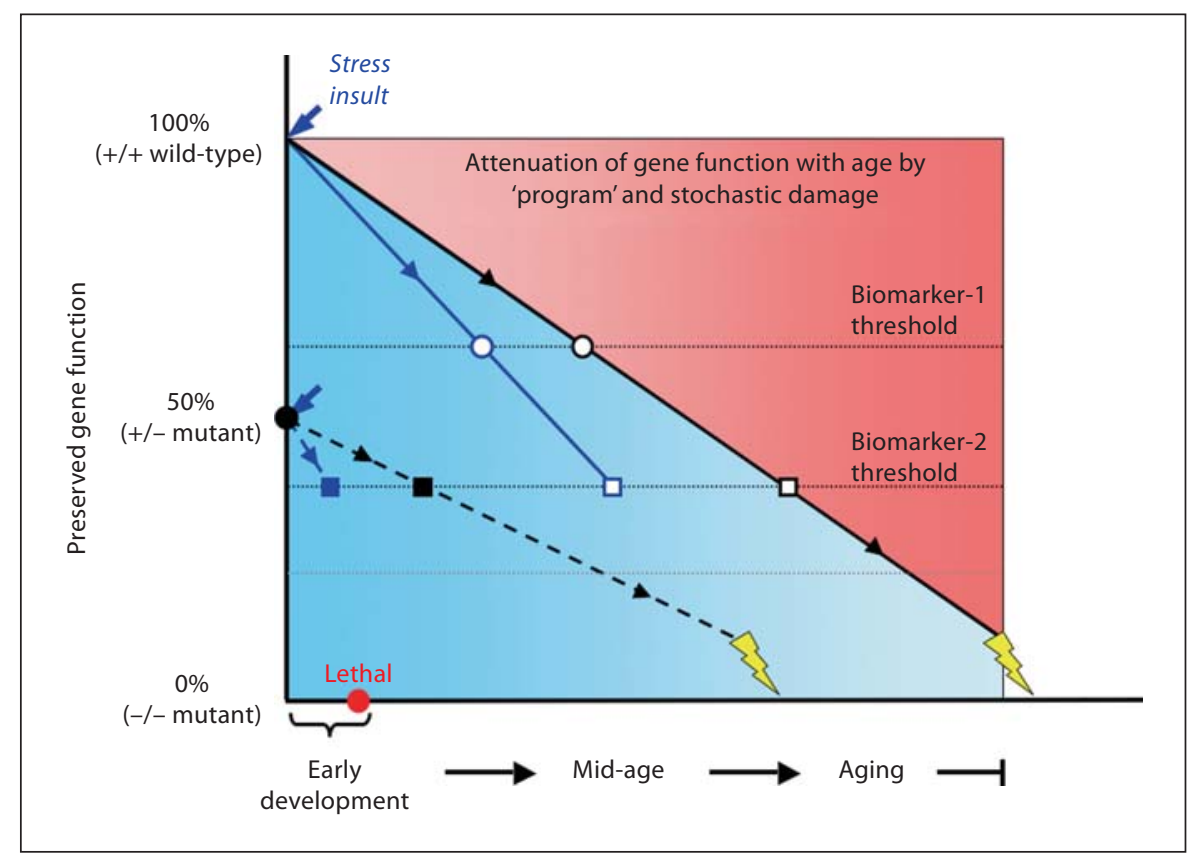

Fig. 5. Gene mutation may result in accelerated decline in gene function and reveal biomarkers of aging earlier in life: a process facilitated by stressors. In this schematic, the slope of the solid black line illustrates age-dependent regression of accuracy in gene function in a wild-type $(+/+)$ organism due to, in part, 'programmed' senescence, accumulated structural cellular damage following intrinsic and extrinsic (environmental) stochastic events, desynchronization of molecular mechanisms, etc. The slope of the dashed black line shows the age-dependent decline of accuracy in gene function in a heterozygous $(+/-)$ mutant organism identified in our screening, i.e., nrs or terf 2 mutant, with presumed $50 \%$ loss of original gene function at birth leading to a shorter life span (yellow lightening bolt on $\mathrm{x}$-axis). Dotted horizontal lines depict the level of gene function at which certain 'aging biomarkers' can be documented, i.e., aging biomarker thresholds. Blue lines illustrate the more rapid decline of accuracy in the function of the same gene following early exposure to stress in wildtype or a heterozygous mutant organisms. According to this model, in wild-type organisms, aging biomarker-1 manifests around middle age (mid-age) (black open circle). In the heterozygous mutant, this aging biomarker-1 manifests spontaneously in early development without stress insult (solid black circle on y-axis), since its threshold lies above the $50 \%$ level of gene function, thus predicting an accelerated aging phenotype. In contrast, aging biomarker2 is characteristic of old age in a wild-type organism (open black square) and manifests in heterozygous mutant during relatively

Although the other nine genes linked to increased SA$\beta$-gal activity during embryogenesis await complete characterization at mature stages (fig. 6), they are of significant interest for their potential involvement in aging, related to neurological functions. A brief description of early adulthood even before mid-age (solid black square). Thus, neither organism would show this biomarker spontaneously during early development. However, in both organisms, exposure to stress (e.g. oxidative stress or $\gamma$-radiation that we used in our studies) during development or later in life can change the dynamics of gene function with age, presumably through additional structural and functional damage to cells. As a result, after stress, both aging biomarkers manifest earlier and the aging biomarker-2 (square) can now be detected during early development of the heterozygous mutant but not the wild-type organism. In homozygous (-/-) mutant organism, either aging biomarker-1 or -2 can be detected during early development and the animals are lethal (solid red circle on x-axis). Thus, the search for gene mutations that might lead to accelerated aging phenotypes can be conducted during early development of homozygous mutants as well as heterozygous mutants with or without the use of environmental stress factors. Note that while this highly simplified schematic illustrates changes that helped us to predict accelerated aging in zebrafish mutants with altered $n r s$ and terf 2 genes, it does not reflect the more complex relationships between the dynamics of gene functions and age, that would be predicted by the 'antagonistic pleiotropy' theory of aging $[30,72]$. Moreover, the concerted effect of numerous genes functioning in parallel throughout life would predictably cause the overall aging process to exhibit non-linear dynamics, with stochastic environmental factors providing further modifications of age-dependent processes under real-life conditions. 
Fig. 6. Retrovirus-insertional mutants showing high SA- $\beta$-gal activity. The eleven homozygous 3.5 days post-fertilization retrovirus-insertional mutants stained with high SA- $\beta$-gal are shown in comparison with a wild-type control (with 1-phenyl-2-thiourea). All of the information we obtained regarding these mutants is summarized in the table of our original publication [18].

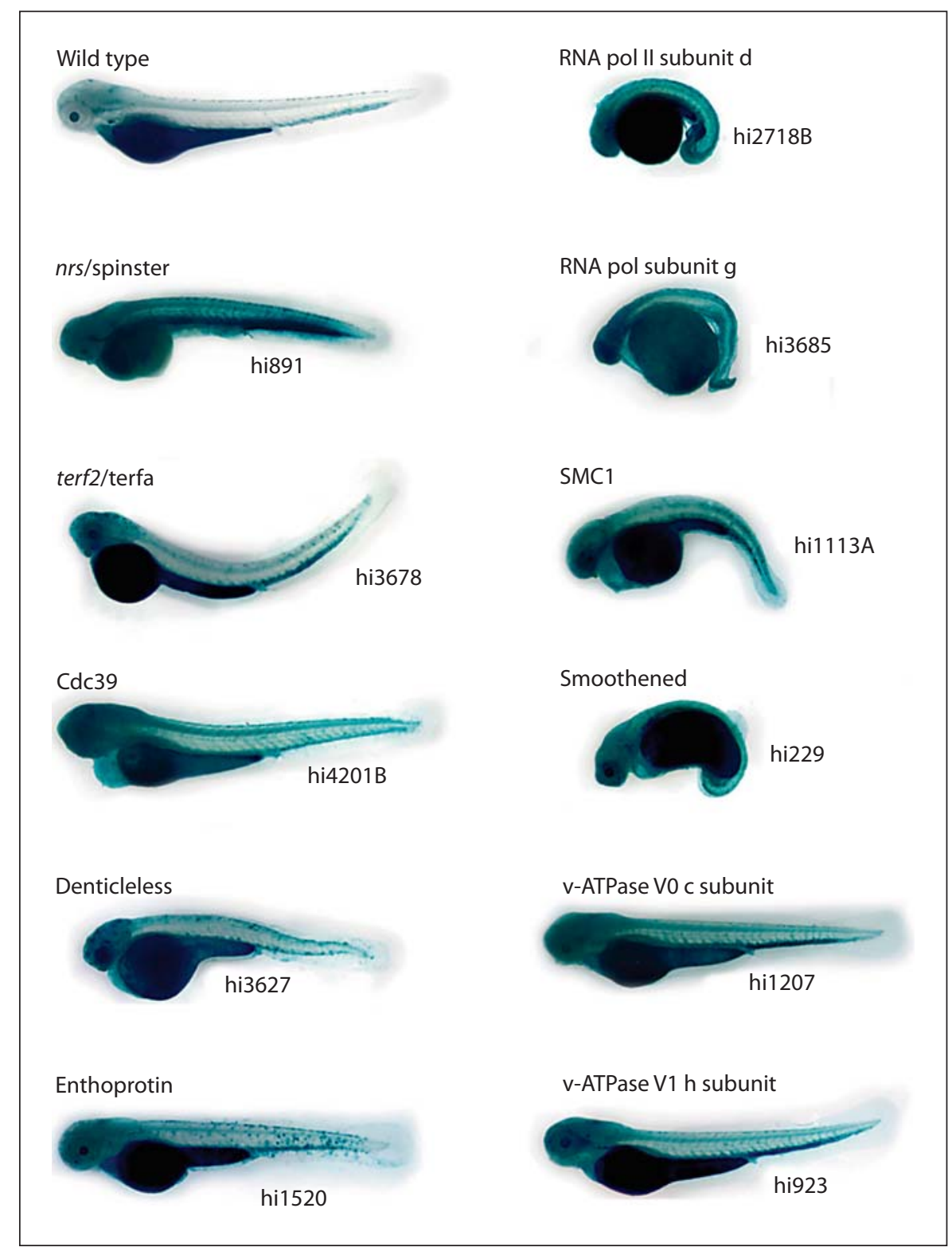

and Scottish subjects [42] and in two Latin-American populations [43], though not in a Japanese population [44]. The role of enthoprotin in regulating the transport and stability of clathrin-coated vesicles, and its enrichment in brain, suggests that it might be important for recycling of synaptic vesicles [45-47]. Thus, impairment in the function of this protein might be associated with degraded cognitive performance.

Vacuolar ATPase. Two of our identified mutant genotypes have alterations in subunits $\mathrm{v} 0 \mathrm{c}$ and $\mathrm{vl} h$ of vacuolar ATPase (v-ATPase). It has been reported that inhibition of the ATP-driven proton pump in RPE lysosomes by the major lipofuscin fluorophore A2-E may contribute to the pathogenesis of AMD [48]. v-ATPase is expressed in rat brain, and its expression levels reportedly increase with age in rat frontal cortex, but not in other areas [49, 50]. In contrast, expression of several subunits of the pump is decreased in aged human cortex [27]. In cortical neuronal cultures, downregulation of v-ATPase expression inhibited cell survival $[49,50]$. Interestingly, the activity of v-ATPase in synaptic vesicles is inhibited by hydrogen peroxide $\left(\mathrm{H}_{2} \mathrm{O}_{2}\right)$, which is produced by activated microglia in brain in neurodegenerative diseases [51]. This leads to a decrease in neurotransmitter uptake into 
synaptic vesicles. Moreover, the v-ATPase V0 subunit a1 is required for a late step in synaptic vesicle exocytosis in Drosophila [52], and also plays an essential role in microglial-mediated neuronal degeneration in zebrafish [53]. The reduction in v-ATPase expression in aging human brain could further erode neurotransmitter uptake, as well as neuron survival, and thus impair cognitive function.

RNA Polymerase II. Our screen identified two genes encoding subunits $\mathrm{D}$ and $\mathrm{G}$ of RNA polymerase II. It is well known that Pol II catalyzes the transcription of DNA to synthesize precursors of mRNA, snRNA, and microRNA [54-56]. Hyperphosphorylation and subcellular mislocation of Pol II was reported in Alzheimer's disease brains, and it was suggested that this may contribute to decreased mRNA synthesis and neuronal degeneration in this disorder [57]. Moreover, both snRNA, and microRNA are believed to play critical roles in neurogenesis and neurodegeneration [58-60].

Smoothened. The G protein-coupled receptor Smoothened (Smo) is activated by the signaling protein Sonic hedgehog (Shh) [61], and plays an essential role in brain development. It is also present in adult brain [62], and recent evidence suggests that Shh, acting through Smo, may be important for the expansion and establishment of postnatal hippocampal neural progenitors [63].

$C d c 39$. This protein, also known as Not1, is a negative regulator of transcription in yeast [64]. Functional characterization of Cdc39 in vertebrates has not yet been performed in detail. However, based on our original observation that Cdc39 mutant fish exhibit small eyes and brains early in development, effects of this mutation on neural and behavioral functions are anticipated.

SMC1. An evolutionarily conserved chromosomal protein, SMC1 (structural maintenance of chromosome protein (1) is a component of the cohesin complex, which is necessary for sister chromatid cohesion. SMC1 is part of the DNA damage response network that functions as an effector in the ATM (ataxia telangiectasia mutated)/ NBS1 (Nijmegen breakage syndrome 1)-dependent cellcycle (S-phase) checkpoint pathway in response to DNA damage $[65,66]$. Ataxia telangiectasia $(A-T)$ is a rare inherited disease showing pleiotropic symptoms including neurodegeneration and premature aging $[67,68]$. Most recently, it was shown that $\mathrm{SMC1}$ functions in postmitotic neurons as a key regulator of axon pruning and neuronal morphogenesis in Drosophila [69].

Denticleless. A regulator of the cell cycle, denticleless (DTL) plays an essential role in the early radiation-induced G2/M checkpoint in both zebrafish and human cells [70]. DTL is a component of the E3-ubiquitin ligase, CUL4A-DDB1 complex in human cells, and is required for CDT1 downregulation during the $S$ phase and following DNA damage. CDT1 is necessary for the formation of the pre-replication complexes that mark sites of DNA replication, and must be degraded during $S$ phase to ensure that the genome is replicated only once per cell cycle [71]. Loss of DTL causes an array of cell-cycle phenotypes in non-irradiated cells, including re-replication and a delay in the $\mathrm{G} 2$ phase resulting from failure to inhibit CDT1 during the $S$ phase. Although the role of DTL in vertebrate neurons has not been characterized, the central nervous system necrotic phenotype in homozygous mutant embryos suggests that a detailed investigation of this mutant would prove fruitful.

The fact that these interesting mutants were selected based on increased SA- $\beta$-gal activity during early development, while no apparent abnormalities were found in heterozygous individuals early on, whereas at least two of them show an accelerated aging phenotype later in life, underscores the utility of such an approach for identifying early predictors of premature or altered aging. This suggests that haploinsufficiency in some genes might not be detrimental to a developing embryo but would facilitate alterations in molecular mechanisms and physiological functions characteristic of aging. If biomarkers of such deficiencies could be documented at an early age, they could help to predict premature aging phenotypes later in life.

Moreover, our studies demonstrate that the same environmental challenges that can accelerate aging in adult zebrafish, e.g. $\gamma$-irradiation $[11,15,16]$, or oxidative stress [pers. unpubl. observation], can be effective in revealing biomarkers of aging during early development. If the coupling of stress responses in zebrafish embryos and/or larvae with aging mechanisms in adult fish holds true, as has been demonstrated in other animal systems and suggested by our results discussed here, this approach will be a useful and powerful tool in the search for aging-related genes. Such speedy identification of genes relevant to vertebrate aging using embryos or larvae would partially circumvent the need for time-consuming and lengthy life span analyses in all available zebrafish mutants.

\section{Conclusion}

Together the results of our studies suggest that aging in zebrafish is associated with processes similar to those observed in aged humans, which manifest on the cellular 
and organismal levels. These include morphological and histological changes in multiple tissues, reduced regeneration and damage to macromolecules (DNA, proteins, and lipids), lipofuscin accumulation possibly resulting in reduced intracellular trafficking, cognitive decline, circadian dysregulation and sleep alterations. The advantages of using zebrafish as a model to explore the mechanisms of human aging and to search for prophylactic and treatment strategies include the fact that both species are diurnal vertebrates with gradual senescence. Moreover, the small size, high reproductive rate, and external development of a transparent embryo make zebrafish uniquely suitable for use in unbiased screens for genes involved in accelerated or delayed aging.

Aging has been thought of as a stochastically 'deteriorative/destructive' process, whereas development is a finely 'programmed/constructive' process. However, the genetic robustness of aging may already be defined during early developmental stages. For example, a certain gene mutation might lead to accelerated decline in gene function that would normally occur only at old age, and this process could be facilitated by stressors. In such a case, the biomarker of aging could be potentially revealed at a much earlier age, as schematically illustrated in figure 5.

As we have shown, this issue can be fruitfully explored using unbiased genetic mutant screens for senescence-associated biomarkers during early developmental stages [18]. Overall, we suggest that the complexity of aging in higher organisms, involving interaction of multiple genes and signaling pathways, warrants a comprehensive search for early predictors of altered aging phenotypes later in life. The zebrafish animal model, with its well-established advantages of forward genetics and improved highthroughput technologies, offers an unparalleled opportunity to identify aging-related genes, and to analyze their function throughout life.

These advantages do not negate the obvious limitations of zebrafish, a non-mammalian aquatic and poikilothermic vertebrate, in addressing some of the processes characteristic of human aging. However, the highly conserved mechanisms of aging on the cellular, tissue and organismal level can and should be addressed using zebrafish as the most affordable genetic model in vertebrates, comparable to Drosophila and C. elegans in invertebrates.

\section{Acknowledgements}

We are very grateful to members of the Kishi and Zhdanova laboratories for their technical assistance. We acknowledge Eriko Koshiizu, Peter E. Bayliss, Shintaro Imamura, Lili Yu, and Marcos A. Lopez-Patino. We thank Christopher Carr for helpful comments. We also thank Shota Shunsuke and Junko Kishi for reading of the manuscript. This work is funded by research grants from the A-T Children's Project, the Ellison Medical Foundation and NIA/NIH to S.K., and from NIMH/NIH and the ChaikinWile Foundation to I.V.Z.

\section{References}

1 Herbig U, Ferreira M, Condel L, Carey D, Sedivy JM: Cellular senescence in aging primates. Science 2006;311:1257.

2 Hoffman KL, McNaughton BL: Coordinated reactivation of distributed memory traces in primate neocortex. Science 2002;297:20702073.

- 3 Francis PJ, Appukuttan B, Simmons E, Landauer N, Stoddard J, Hamon S, Ott J, Ferguson B, Klein M, Stout JT, Neuringer M: Rhesus monkeys and humans share common susceptibility genes for age-related macular disease. Hum Mol Genet 2008; 17 : 2673-2680.

4 Peters A: Structural changes in the normally aging cerebral cortex of primates. Prog Brain Res 2002;136:455-465.

5 Hasty P, Campisi J, Hoeijmakers J, van Steeg $\mathrm{H}$, Vijg J: Aging and genome maintenance: lessons from the mouse? Science 2003;299: 1355-1359.
6 Smale L, Lee T, Nunez AA: Mammalian diurnality: some facts and gaps. J Biol Rhythms 2003;18:356-366.

7 Kondratov RV, Kondratova AA, Gorbacheva VY, Vykhovanets OV, Antoch MP: Early aging and age-related pathologies in mice deficient in BMAL1, the core component of the circadian clock. Genes Dev 2006;20:18681873.

$\checkmark 8$ McDearmon EL, Patel KN, Ko CH, Walisser JA, Schook AC, Chong JL, Wilsbacher LD, Song EJ, Hong HK, Bradfield CA, Takahashi JS: Dissecting the functions of the mammalian clock protein BMAL1 by tissue-specific rescue in mice. Science 2006;314:13041308.

-9 Doi M, Hirayama J, Sassone-Corsi P: Circadian regulator CLOCK is a histone acetyltransferase. Cell 2006;125:497-508.

10 Kondratov RV: A role of the circadian system and circadian proteins in aging. Ageing Res Rev 2007;6:12-27.
1 Kishi S, Uchiyama J, Baughman AM, Goto T, Lin MC, Tsai SB: The zebrafish as a vertebrate model of functional aging and very gradual senescence. Exp Gerontol 2003;38: 777-786.

12 Keller ET, Murtha JM: The use of mature zebrafish (Danio rerio) as a model for human aging and disease. Comp Biochem Physiol C Toxicol Pharmacol 2004;138:335-341.

13 Gerhard GS, Kauffman EJ, Wang X, Stewart R, Moore JL, Kasales CJ, Demidenko E, Cheng KC: Life spans and senescent phenotypes in two strains of zebrafish (Danio rerio). Exp Gerontol 2002;37:1055-1068.

14 Zhdanova IV, Yu L, Lopez-Patino M, Shang E, Kishi S, Guelin E: Aging of the circadian system in zebrafish and the effects of melatonin on sleep and cognitive performance. Brain Res Bull 2008;75:433-441.

15 Yu L, Tucci V, Kishi S, Zhdanova IV: Cognitive aging in zebrafish. PLoS ONE 2006;1: e14. 
-16 Tsai SB, Tucci V, Uchiyama J, Fabian NJ, Lin MC, Bayliss PE, Neuberg DS, Zhdanova IV, Kishi S: Differential effects of genotoxic stress on both concurrent body growth and gradual senescence in the adult zebrafish. Aging Cell 2007;6:209-224.

$\checkmark 17$ Lieschke GJ, Currie PD: Animal models of human disease: zebrafish swim into view. Nat Rev Genet 2007;8:353-367.

-18 Kishi S, Bayliss PE, Uchiyama J, Koshimizu E, Qi J, Nanjappa P, Imamura S, Islam A, Neuberg D, Amsterdam A, Roberts TM: The identification of zebrafish mutants showing alterations in senescence-associated biomarkers. PLoS Genet 2008;4:e1000152.

$>19$ Jung T, Bader N, Grune T: Lipofuscin: formation, distribution, and metabolic consequences. Ann NY Acad Sci 2007;1119:97111.

20 Schmucker DL: Age-related changes in liver structure and function: implications for disease? Exp Gerontol 2005;40:650-659.

-21 Poleo G, Brown CW, Laforest L, Akimenko MA: Cell proliferation and movement during early fin regeneration in zebrafish. Dev Dyn 2001;221:380-390.

-22 Murtha JM, Keller ET: Characterization of the heat shock response in mature zebrafish (Danio rerio). Exp Gerontol 2003;38:683691.

-23 Keller JM, Escara-Wilke JF, Keller ET: Heat stress-induced heat shock protein 70 expression is dependent on ERK activation in zebrafish (Danio rerio) cells. Comp Biochem Physiol A Mol Integr Physiol 2008; 150:307314.

-24 Malek RL, Sajadi H, Abraham J, Grundy MA, Gerhard GS: The effects of temperature reduction on gene expression and oxidative stress in skeletal muscle from adult zebrafish. Comp Biochem Physiol C Toxicol Pharmacol 2004;138:363-373.

25 Zhdanova IV, Wang SY, Leclair OU, Danilova NP: Melatonin promotes sleep-like state in zebrafish. Brain Res 2001;903:263-268.

26 Zhdanova IV: Melatonin as a hypnotic: pro. Sleep Med Rev 2005;9:51-65.

-27 Lu T, Pan Y, Kao SY, Li C, Kohane I, Chan J, Yankner BA: Gene regulation and DNA damage in the ageing human brain. Nature 2004;429:883-891.

$\checkmark 28$ Contestabile A, Ciani E: The place of choline acetyltransferase activity measurement in the 'cholinergic hypothesis' of neurodegenerative diseases. Neurochem Res 2008;33: 318-327.

29 Bartus RT, Dean RL 3rd, Beer B, Lippa AS: The cholinergic hypothesis of geriatric memory dysfunction. Science 1982;217:408-414.

-30 Martin GM: Modalities of gene action predicted by the classical evolutionary biological theory of aging. Ann NY Acad Sci 2007a;1100:14-20.

-31 Martin GM, Bergman A, Barzilai N: Genetic determinants of human health span and life span: progress and new opportunities. PLoS Genet 2007b;3:e125.
2 Amsterdam A, Nissen RM, Sun Z, Swindell EC, Farrington S, Hopkins N: Identification of 315 genes essential for early zebrafish development. Proc Natl Acad Sci USA 2004; 101:12792-12797.

33 Dimri GP, Lee X, Basile G, Acosta M, Scott G, Roskelley C, Medrano EE, Linskens M, Rubelj I, Pereira-Smith O, et al: A biomarker that identifies senescent human cells in culture and in aging skin in vivo. Proc Natl Acad Sci USA 1995;92:9363-9367.

34 Cao L, Li W, Kim S, Brodie SG, Deng CX: Senescence, aging, and malignant transformation mediated by p53 in mice lacking the Brcal full-length isoform. Genes Dev 2003; 17:201-213.

35 Keyes WM, Wu Y, Vogel H, Guo X, Lowe SW, Mills AA: 63 deficiency activates a program of cellular senescence and leads to accelerated aging. Genes Dev 2005;19:1986-1999.

- 36 Valenzano DR, Terzibasi E, Cattaneo A, Domenici L, Cellerino A: Temperature affects longevity and age-related locomotor and cognitive decay in the short-lived fish Nothobranchius furzeri. Aging Cell 2006;5:275278.

37 Kishi S: Functional aging and gradual senescence in zebrafish. Ann NY Acad Sci 2004; 1019:521-526.

38 Kishi S: Zebrafish as aging models; in Conn M (ed): Handbook of Models for Human Aging. New York, Elsevier Academic Press, 2006, pp 317-338.

39 Nakano Y, Fujitani K, Kurihara J, Ragan J, Usui-Aoki K, Shimoda L, Lukacsovich T, Suzuki K, Sezaki M, Sano Y, Ueda R, Awano W, Kaneda M, Umeda M, Yamamoto D: Mutations in the novel membrane protein spinster interfere with programmed cell death and cause neural degeneration in Drosophila melanogaster. Mol Cell Biol 2001;21:37753788.

40 Celli GB, de Lange T: DNA processing is not required for ATM-mediated telomere damage response after TRF2 deletion. Nat Cell Biol 2005;7:712-718.

41 Van Steensel B, Smogorzewska A, de Lange T: TRF2 protects human telomeres from end-to-end fusions. Cell 1998;92:401-413.

42 Pimm J, McQuillin A, Thirumalai S, Lawrence J, Quested D, Bass N, Lamb G, Moorey H, Datta SR, Kalsi G, Badacsonyi A, Kelly K, Morgan J, Punukollu B, Curtis D, Gurling H: The Epsin 4 gene on chromosome 5q, which encodes the clathrin-associated protein enthoprotin, is involved in the genetic susceptibility to schizophrenia. Am J Hum Genet 2005;76:902-907.

43 Herzberg I, Jasinska A, Garcia J, Jawaheer D, Service S, Kremeyer B, Duque C, Parra MV, Vega J, Ortiz D, Carvajal L, Polanco G, Restrepo GJ, Lopez C, Palacio C, Levinson M, Aldana I, Mathews C, Davanzo P, Molina J, Fournier E, Bejarano J, Ramirez M, Ortiz CA, Araya X, Sabatti C, Reus V, Macaya G, Bedoya G, Ospina J, Freimer N, Ruiz-Linares A: Convergent linkage evidence from two
Latin-American population isolates supports the presence of a susceptibility locus for bipolar disorder in 5q31-34. Hum Mol Genet 2006;15:3146-3153.

-44 Richards M, Iijima Y, Shizuno T, Kamegaya Y, Hori H, Omori M, Arima K, Saitoh O, Kunugi H: Failure to confirm an association between Epsin 4 and schizophrenia in a Japanese population. J Neural Transm 2008;115: 1347-1354.

45 Wasiak S, Legendre-Guillemin V, Puertollano R, Blondeau F, Girard M, de Heuvel E, Boismenu D, Bell AW, Bonifacino JS, McPherson PS: Enthoprotin: a novel clathrin-associated protein identified through subcellular proteomics. J Cell Biol 2002;158: 855-862.

46 Wasiak S, Denisov AY, Han Z, Leventis PA, de Heuvel E, Boulianne GL, Kay BK, Gehring K, McPherson PS: Characterization of a $\gamma$-adaptin ear-binding motif in enthoprotin. FEBS Lett 2003;555:437-442.

47 McPherson PS, Ritter B: Peptide motifs: building the clathrin machinery. Mol Neurobiol 2005;32:73-87.

48 Bergmann M, Schutt F, Holz FG, Kopitz J: Inhibition of the ATP-driven proton pump in RPE lysosomes by the major lipofuscin fluorophore A2-E may contribute to the pathogenesis of age-related macular degeneration. FASEB J 2004; 18:562-564.

49 Numata M, Ohkuma S, Iseki S: Expression and localization of mRNA encoding $16 \mathrm{kDa}$ subunit of vacuolar $\mathrm{H}^{+}$-ATPase in rat brain. Cell Biol Int 1995;19:1-7.

50 Hung H, Tsai MJ, Wu HC, Lee EH: Age-dependent increase in C7-1 gene expression in rat frontal cortex. Brain Res Mol Brain Res 2000;75:330-336.

51 Wang Y, Floor E: Hydrogen peroxide inhibits the vacuolar $\mathrm{H}^{+}$-ATPase in brain synaptic vesicles at micromolar concentrations. J Neurochem 1998;70:646-652.

52 Hiesinger PR, Fayyazuddin A, Mehta SQ, Rosenmund T, Schulze KL, Zhai RG, Verstreken P, Cao Y, Zhou Y, Kunz J, Bellen HJ: The v-ATPase V0 subunit a1 is required for a late step in synaptic vesicle exocytosis in Drosophila. Cell 2005;121:607-620.

53 Peri F, Nüsslein-Volhard C: Live imaging of neuronal degradation by microglia reveals a role for v0-ATPase a1 in phagosomal fusion in vivo. Cell 2008;133:916-927.

54 Cramer P, Armache KJ, Baumli S, Benkert S, Brueckner F, Buchen C, Damsma GE, Dengl S, Geiger SR, Jasiak AJ, Jawhari A, Jennebach S, Kamenski T, Kettenberger H, Kuhn CD, Lehmann E, Leike K, Sydow JF, Vannini A: Structure of eukaryotic RNA polymerases. Annu Rev Biophys 2008;37:337-352.

55 Lee Y, Kim M, Han J, Yeom KH, Lee S, Baek SH, Kim VN: MicroRNA genes are transcribed by RNA polymerase II. EMBO J 2004;23:4051-4060.

56 Egloff S, O'Reilly D, Murphy S: Expression of human snRNA genes from beginning to end. Biochem Soc Trans 2008;36:590-594. 
-57 Husseman JW, Hallows JL, Bregman DB, Leverenz JB, Nochlin D, Jin LW, Vincent I: Hyperphosphorylation of RNA polymerase II and reduced neuronal RNA levels precede neurofibrillary tangles in Alzheimer disease. J Neuropathol Exp Neurol 2001;60: 1219-1232.

58 Giraldez AJ, Cinalli RM, Glasner ME, Enright AJ, Thomson JM, Baskerville S, Hammond SM, Bartel DP, Schier AF: MicroRNAs regulate brain morphogenesis in zebrafish. Science 2005;308:833-838.

-59 Schaefer A, O'Carroll D, Tan CL, Hillman D, Sugimori M, Llinas R, Greengard P: Cerebellar neurodegeneration in the absence of $\mathrm{mi}-$ croRNAs. J Exp Med 2007;204:1553-1558.

60 Winkler C, Eggert C, Gradl D, Meister G, Giegerich M, Wedlich D, Laggerbauer B, Fischer U: Reduced U snRNP assembly causes motor axon degeneration in an animal model for spinal muscular atrophy. Genes Dev 2005;19:2320-2330.

61 Zhu G, Mehler MF, Zhao J, Yu Yung S, Kessler JA: Sonic hedgehog and BMP2 exert opposing actions on proliferation and differentiation of embryonic neural progenitor cells. Dev Biol 1999;215:118-129.
62 Charytoniuk D, Porcel B, Rodriguez Gomez J, Faure H, Ruat M, Traiffort E: Sonic hedgehog signalling in the developing and adult brain. J Physiol Paris 2002;96:9-16.

63 Han YG, Spassky N, Romaguera-Ros M, Garcia-Verdugo JM, Aguilar A, SchneiderMaunoury S, Alvarez-Buylla A: Hedgehog signaling and primary cilia are required for the formation of adult neural stem cells. Nat Neurosci 2008;11:277-284.

64 Collart MA, Struhl K: NOT1(CDC39), NOT2(CDC36), NOT3, and NOT4 encode a global-negative regulator of transcription that differentially affects TATA-element utilization. Genes Dev 1994;8:525-537.

65 Yazdi PT, Wang Y, Zhao S, Patel N, Lee EY, Qin J: SMC1 is a downstream effector in the ATM/NBS1 branch of the human S-phase checkpoint. Genes Dev 2002;16:571-582.

66 Kim ST, Xu B, Kastan MB: Involvement of the cohesin protein, Smcl, in Atm-dependent and independent responses to DNA damage. Genes Dev 2002;16:560-570.
67 Barzilai A, Rotman G, Shiloh Y: ATM deficiency and oxidative stress: a new dimension of defective response to DNA damage. DNA Repair (Amst) 2002;1:3-25.

68 Biton S, Barzilai A, Shiloh Y: The neurological phenotype of ataxia-telangiectasia: solving a persistent puzzle. DNA Repair (Amst) 2008;7:1028-1038.

69 Schuldiner O, Berdnik D, Levy JM, Wu JS, Luginbuhl D, Gontang AC, Luo L: piggyBacbased mosaic screen identifies a postmitotic function for cohesin in regulating developmental axon pruning. Dev Cell 2008;14:227238

70 Sansam CL, Shepard JL, Lai K, Ianari A, Danielian PS, Amsterdam A, Hopkins N, Lees JA: DTL/CDT2 is essential for both CDT1 regulation and the early G2/M checkpoint. Genes Dev 2006;20:3117-3129.

71 Bell SP, Dutta A: DNA replication in eukaryotic cells. Annu Rev Biochem 2002;71:333374 .

72 Williams PD, Day T: Antagonistic pleiotropy, mortality source interactions, and the evolutionary theory of senescence. Evolution 2003;57:1478-1488. 